\title{
“PRIMARIZAÇÃO” DA EXECUÇÃO DE MANUTENÇÃO NA ÁREA DE ENERGIA DA ARCELORMITTAL AÇOS PLANOS AMÉRICA DO SUL*
}

\author{
Humberto Celeste Gomes ${ }^{1}$ \\ Adilson Campo Ferrarini \\ Eduardo Luiz Sales Marinho ${ }^{3}$ \\ João Victor de Carvalho Bomfim ${ }^{4}$
}

\section{Resumo}

Este trabalho irá apresentar os critérios e considerações que levaram a AMT decidir pela primarização de duas equipes de manutenção da área de Energia (equipe de manutenção mecânica das turbomáquinas e equipe de manutenção elétrica de SEP/RUG). Apresentará também os desafios de formação, treinamento e capacitação das equipes bem como abordará a avaliação dos ganhos do projeto. Ganho em produtividade: Aumento da produtividade da equipe com a redução de $11 \%$ da quantidade total de empregados e Ganho financeiro para a empresa em função de redução de custo fixo. Ganhos adicionais: Melhoria na qualidade dos serviços, com menor retrabalho e turn over; oportunidade de redução de contratação SPOT em outras atividades envolvendo equipamentos rotativos de menor porte (ex: bombas); Formação de uma base de técnicos para futuras promoções internas (inspetores, técnicos de manutenção dentre outras). Pontos de atenção: Serviços realizados fora do horário ADM pela equipe de manutenção; Formação de turno de revezamento na equipe de manutenção de Turbomáquinas em grandes paradas.

Palavras-chave: Primarização; Ganho; Redução FTE.

\section{Abstract}

INSOUSING OF ENERGY AREA MAINTENANCE EXECUTION OF ARCELORMITTAL FLAT CARBON SOUTH AMERICA

This paper will present the criterion and considerations that made AMT decide to insourcing two teams of maintenance of Energy area (mechanical maintenance team of turbomachinery and electrical maintenance team of SEP/RUG). Will also present the challenges of formation, professional training and qualification of the teams as well as show the project's benefits. Productivity gain: Reduction of the total number of employees reduction of $11 \%$; Financial gain to the company because of the fixed cost reduction. Aditional gain: Improvement of service quality, reduction of turn over and execution of the same job; Opportunity of reduction of SPOT hiring in other activities involving small size rotational equipment (i.e. pumps); Formation of technical base for future internal promotion (inspectors, maintenance technicist amongst others). Attention topics: Work done by maintenance team out of work hour; Formation of switching turns of maintenance team of turbomachinery in big stops.

Keywords: Insourcing; Profit; Reduction FTE.

Engenheiro mecânico, Gerente de operação de Coqueria, IGCP / IGC, ArcelorMittal Tubarão, Serra, ES, Brasil.

2 Engenheiro mecânico, Gerente de manutenção da área de energia, IGEG / IGE, ArcelorMittal Tubarão, Serra, ES, Brasil

3 Engenheiro mecânico, especialista em manutenção mecânica, área de energia IGE, ArcelorMittal Tubarão, ES, Brasil.

4 Engenheiro mecânico, especialista em manutenção mecânica, área de energia IGE, ArcelorMittal Tubarão, ES, Brasil. 


\section{INTRODUÇÃO}

A terceirização foi adotada pelas empresas brasileiras com o objetivo de reduzir custos de mão de obras devido à competitividade do mercado. Porém com o passar do tempo, percebeu-se que existia alguns pontos negativos como falta qualificação da mão-de-obra gerando baixa qualidade, a diminuição do vínculo e do comprometimento profissional, alta rotatividade da mão-de-obra e baixa produtividade.

Então, foi adotado o processo de primarização e análises mostraram que iriamos ter ganhado com redução de FTE e custo, recuperação do "know how" dos equipamentos, aumento da produtividade, qualidade e redução de retrabalhos, redução de contingências trabalhistas, formação de reserva técnica para promoções.

\section{MATERIAIS E MÉTODOS}

\section{1 - Na Área de Energia da ArcelorMittal Tubarão Foram Primarizados 02 Contratos de Manutenção}

O contrato de manutenção de turbomáquinas que tem os seguintes equipamentos:

- Turbina a vapor e gerador: 06 unidades instaladas (capacidade geração: 486MW);

- Soprador de ar (Alto forno): 04 (potência: 162MW);

- Turbina de recuperação: 01 (capacidade geração: 20MW);

- Compressor centrífugo: 20 (potência: 65MW);

- Turbo bomba: 22 (potência: 28MW);

O contrato de manutenção da rede de distribuição de energia elétrica (SEP / RUG):

- Estações recebedoras: 03 (Tensão: 138KV);

- Transformadores > 1MVA: (23 unidades);

- Subestações elétricas: 17;

- Cabos de transmissão $138 \mathrm{KV}(100 \mathrm{Km})$;

- Cabos de transmissão 34,5KV, 13,8 KV e 3,3 KV (800Km);

- Geradores diesel de emergência: 04;

- Disjuntores e relés de proteção (223 e 4.000 unidades)

\section{2- Contextualização}

Os fatores motivadores que levaram ao estudo dos prós e contras da primarização foram identificados pela área conforme a seguir: 
SITUAÇÃO NO PASSADO

\section{Terceirização}

- Baixo Custo;

- Empresas Especializadas (muitas opções);

- Certificação de Terceiros.

\section{Primarização}

- Alto Custo;

- Foco no Core Business;

- Baixa demanda (parque de ativos fase 1AMT).

Fonte: ArcelorMittal Tubarão

\section{3 - Projeto Primarização da Manutenção}

\subsection{1 - Objetivo}

- Redução de FTE e custos de manutenção;

- Aumentar a produtividade na execução de serviços de manutenção;

- Reduzir retrabalhos nos serviços de manutenção

\subsection{2 - Premissas}

- Considerado efetivo médio mensal das contratadas;

- Sinergia nas equipes de manutenção existentes;

- Considerado a necessidade de complementar as equipes próprias com empresas contratadas e/ou apoio na seguinte oportunidade: Grande parada de CTE (TGS + periféricos caldeira) e SEP (plurianual);

- Considerado aquisição de ferramentas e equipamentos de testes especiais;

- Considerado a locação de recursos de deslocamento e transporte interno.

\subsection{3 - Definição de escopo}

Relação de serviços previstos no contrato da empresa terceirizada e colocado como proposta de fornecimento da equipe primarizada da ArcelorMittal. O exemplo a seguir foi realizado com a empresa de manutenção de turbomaquinas. 
Tabela $\mathrm{n}^{0} \mathbf{1}$

\begin{tabular}{|c|c|c|c|}
\hline \multirow[b]{2}{*}{ Escopo principal } & \multicolumn{2}{|c|}{ Situação Atual } & \multirow{2}{*}{$\begin{array}{c}\text { Situação proposta } \\
\text { AMT }\end{array}$} \\
\hline & $\begin{array}{l}\text { Empresa } \\
\text { manutenção } \\
\text { rotativos }\end{array}$ & AMT & \\
\hline Planejamento macro dos serviços; & $x$ & - & $x$ \\
\hline $\begin{array}{l}\text { Levantamento de dispositivos especiais e } \\
\text { sobressalentes; }\end{array}$ & $\mathrm{x}$ & - & $x$ \\
\hline $\begin{array}{l}\text { Inspeção, conservação e estocagem de } \\
\text { sobressalentes no AXO da AMT; }\end{array}$ & $x$ & - & $\mathrm{X}$ \\
\hline $\begin{array}{l}\text { Aquisição de dispositivos especiais e } \\
\text { sobressalentes; }\end{array}$ & - & $x$ & $\mathrm{X}$ \\
\hline Elaboração de padrão detalhado de manutenção; & $x$ & - & $x$ \\
\hline Emissão de OS de manutenção & - & $\mathrm{X}$ & $\mathrm{X}$ \\
\hline $\begin{array}{l}\text { Execução de manutenção de turbomáquinas, } \\
\text { bombas e compressores; }\end{array}$ & $x$ & - & $x$ \\
\hline $\begin{array}{l}\text { Execução de manutenção em equipamentos } \\
\text { periféricos (tq de óleo, cooler de óleo e retirada de } \\
\text { motores elétricos) }\end{array}$ & $x$ & - & Nota 1 \\
\hline
\end{tabular}

Nota 1 - Serviços não requerem especialização, deverão continuar Fonte: ArcelorMittal Tubarão

\subsection{4 - Cronograma de implantação do projeto}

O cronograma de implantação contempla as etapas de admissão, Treinamento, Infra-estrutura e início da execução conforme a seguir:

\begin{tabular}{|c|c|c|c|c|c|c|c|c|c|c|c|c|}
\hline & & \multicolumn{11}{|c|}{ Meses } \\
\hline CRONOGRAMA MACRO PRIMARIZAÇÁO & Resp & 1 & 2 & 3 & 4 & 5 & 6 & 7 & 8 & 9 & 10 & 11 \\
\hline 1|Admissão & & & & & & & & & & & & \\
\hline 1.1 Levantamento de curriculum vitae & IGEG & & & & & & & & & & & \\
\hline 1.2 Teste Pesicotécnico & FHD & & & & & & & & & & & \\
\hline \begin{tabular}{l|l}
1.3 & Exame médico \\
\end{tabular} & FHS & & & & & & & & & & & \\
\hline \begin{tabular}{l|l|}
1.4 & Entrevista \\
\end{tabular} & IGEG & & & & & & & & & & & \\
\hline \begin{tabular}{l|l|}
1.5 & Admissão \\
\end{tabular} & FHD & & & & & & & & & & & \\
\hline & & & & & & & & & & & & \\
\hline \begin{tabular}{l|l}
2 & Treinamento (PINE) \\
\end{tabular} & & & & & & & & & & & & \\
\hline 2.1 Elaboração do PINE área (foco em turbomáquinas e SEP) & IGEG & & & & & & & & & & & \\
\hline \begin{tabular}{l|l}
2.2 & Treinamento (PINE Institucional)) \\
\end{tabular} & FHD & & & & & & & & & & & \\
\hline \begin{tabular}{l|l}
2.3 & Treinamento (Pine Área) \\
\end{tabular} & IGEG & & & & & & & & & & & \\
\hline \begin{tabular}{l|l}
2.4 & Teste e-learning (verificação do aprendizado) \\
\end{tabular} & IGEG & & & & & & & & & & & \\
\hline \begin{tabular}{l|l|}
2.5 & Repescagem (PINE Área + e-learning) \\
\end{tabular} & IGEG & & & & & & & & & & & \\
\hline $\begin{array}{l}\text { 3Infra-estrutura } \\
\end{array}$ & 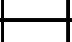 & & & & & & & & & & & \\
\hline 3.1 Escritório (salas, computadores e outros) & IGEG & & & & & & & & & & & \\
\hline 3.2 Aquisição de ferramentas & IGEG & & & & & & & & & & & \\
\hline \begin{tabular}{l|l}
3.3 & PDI (cabines) \\
.
\end{tabular} & IGEG & & & & & & & & & & & \\
\hline \begin{tabular}{l|l|l}
3.4 & Veículo \\
\end{tabular} & IGEG & & & & & & & & & & & \\
\hline \begin{tabular}{l|l}
3.5 & Rádio Comunicação \\
\end{tabular} & IGEG & & & & & & & & & & & \\
\hline \begin{tabular}{|l|l|}
3.6 & Definição dos EPl's \\
\end{tabular} & IGEG & & & & & & & & & & & \\
\hline & & & & & & & & & & & & \\
\hline 4 | Início Execução & IGEG & & & & & & & & & & & \\
\hline & & & & & & & & & & & & \\
\hline 5 Over lap previsto & IGEG & & & & & & & & & & & \\
\hline
\end{tabular}

Fonte: ArcelorMittal Tubarão.

\subsection{5 - Fluxo financeiro do projeto}

A primarização da equipe, prevê um tempo de seis meses para começar a execução, com custos iniciais para compra de ferramentas e instrumentos, 
Admissão de pessoal e salários. A partir do sexto mês começa a execução de serviços com os ganhos financeiros previstos no projeto.

\section{CONCLUSÃO}

3.1 -Ganho em produtividade:

- Redução $11 \%$ pessoal

- Redução de custo de 39\% quando o projeto for finalizado (previsão Q1 de 2014);

3.2 - Desembolsos pontuais do projeto

- Compra de ferramentas e equipamentos;

- Custo com admissão e EPl's;

- Overlap previsto de aprox 03 meses.

3.3 - Ganhos potenciais:

- Jornada para Zero Acidente: melhorias segurança - Cultura AMT;

- Melhoria na qualidade dos serviços, com menor retrabalho e turn over;

- Oportunidade de implantação de melhorias nos métodos de execução de serviços com foco em segurança e produtividade;

- Oportunidade de redução de contratação SPOT em outras atividades envolvendo equipamentos rotativos de menor porte (ex: bombas);

- Formação de equipe especializada na realização de ensaios elétricos em equipamentos de alta e média tensão - Intensificação da manutenção preditiva;

- Formação de uma base de técnicos para futuras promoções internas (inspetores, técnicos de manutenção dentre outras);

\section{4 - Pontos de Atenção:}

- Serviços realizados fora do horário ADM pela equipe de manutenção da RUG;

- $\mathrm{Na}$ equipe de manutenção da RUG a função de motorista será desempenhada por membros da própria equipe primarizada;

- Formação de turno de revezamento na equipe de manutenção de Turbomáquinas em grandes paradas de CTE.

Demais casos são opcionais. 